group might have influenced outcome. With respect to characteristics of the study group, participants in our study manifested both self-poisoning (91\%) and self-injury (9\%) irrespective of the apparent purpose of the act, and therefore can be considered a representative sample of patients who self-harm. Of the contacted participants, only $7.3 \%$ were excluded because of schizophrenia or alcohol and drug misuse. Our final sample consisted of females (94\%) with a long history of self-harm (77\% reported 10 or more previous episodes of self-poisoning and/or self-injury) and severe psychological and psychiatric problems (on average four psychiatric diagnoses (mood and anxiety disorders in particular)). It is possible that CBT as an add-on to TAU is more likely to be effective for people with such chronic and severe self-harm. The fact that rate of withdrawal from CBT amounted to $17 \%$ underscores the feasibility of an intervention tailored to the needs of this particular group.

In conclusion, CBT appears to be an effective adjunct to TAU in chronic self-harm and further research on moderators and mediators of change seems warranted.

Philip Spinhoven, Unit of Clinical Psychology, Leiden University Institute for Psychological Research, and Department of Psychiatry, Leiden University Medical centre, The Netherlands. Email: spinhoven@fsw.leidenuniv.nl; Ella Arensman National Suicide Research Foundation, Cork, Ireland

doi: 10.1192/bjp.193.1.80b

\section{Virtual reality and paranoia}

The use of virtual reality to create a 'laboratory' is promising. As someone who has played computer games and has used the London underground ('tube') trains almost daily for 4 years, I was interested in the observations that those who used the tube regularly were less likely to have persecutory thinking in virtual reality, whereas an experience of playing computer games was a strong predictor of paranoid thinking. ${ }^{1}$

I am not sure whether the observations can be justified by an assumption that the game-playing individuals were reacting because they automatically processed the computer characters as real. The use of a virtual reality environment may have introduced a bias not taken into account just by estimating the duration of game play.

Cognition and automatic thoughts are based on prior experiences. Has this study taken into account how prior gaming experience may affect one's perception to a virtual reality environment, as opposed to a generalised cognition easily translated to the real world? Is there a possibility that the participants automatically processed the environment as being hostile thus making the findings 'a strong predictor of paranoid thinking' only in a virtual world?

The data provided in the paper fail to show the nature of gaming experience these people have had. Is it possible that a person who plays non-violent strategy games, or gambles online, will have a different experience of virtual reality compared with someone who plays first-person shooters where one of the primary objectives of the game would be to survive, keep safe distance and, of course, to 'kill' other players when they are in range? Also, would the findings be different if some of these people who played computer games spent their time in virtual reality social networking worlds such as 'Second Life'?

If an experience of travelling on the tube regularly shows less likelihood of feeling persecuted in a virtual train ride, can it be said that a prior experience of a threatening virtual reality environment make those who play games more likely to feel persecuted in the chosen medium than they would otherwise be in the real life?

1 Freeman D, Pugh K, Antley A, Slater M, Bebbington P, Gittins M, Dunn G, Kuipers $E$, Fowler D, Garety $P$. Virtual reality study of paranoid thinking in the general population. Br J Psychiatry 2008; 192: 258-63.
Sunanda Ghosh, Hertfordshire Partnership Foundation Trust, UK. Email: sunanda ghosh@gmail.com

doi: 10.1192/bjp.193.1.81

Freeman et al have used an innovative technique in a non-clinical population to confirm a high background prevalence of negative, mistrustful and fearful thoughts about others. ${ }^{1}$ Their paper may be helpful in encouraging healthcare professionals in their attempts to normalise rather than medicalise such thoughts, which are particularly common and pronounced in patients with neurotic and personality disorders. ${ }^{2}$

I am concerned, however, by the authors' use of the word 'paranoia' to describe these thoughts. Freeman et al define paranoia as 'the unfounded fear that others intend to cause you harm', with reference only to an earlier publication by the main author; later in the paper the words 'persecutory' and 'paranoid' are used synonymously. This definition and usage are erroneous.

Varying definitions of paranoia exist in the literature but the correct meaning of 'paranoid' is 'delusional.' ${ }^{3}$ With a Greek derivation and a literal meaning of 'out of the mind', German psychiatrists revived the term in the mid-19th century to describe conditions characterised by delusions, not only of persecution but also of grandeur. ${ }^{4}$ Later, Kraepelin, Bleuler and others variously attempted to classify paranoia, but central to all concepts was that it referred only to delusional rather than non-delusional ideation, and could include grandiose, jealous or somatic, as well as persecutory, delusions. ${ }^{4}$ Indeed, the 'paranoid' subtype of schizophrenia, still in use, refers to an illness dominated by hallucinations and delusions, and the latter need not be persecutory in nature. $^{5}$

Of course, over the 20th century, the word has taken on an entirely different meaning outside psychiatry. Anecdotally, patients frequently report 'paranoia' as an unpleasant presenting complaint, despite the fact that, by its very nature, a fixed false belief cannot be viewed by its sufferer as a symptom. Similarly, mental health professionals commonly use the term erroneously, sometimes resulting in non-psychotic patients being inappropriately referred to specialist services for those with psychosis. I fear that Freeman et al's rejection of the longstanding psychiatric definition of paranoia, in favour of its lay meaning, will only add to this unnecessary confusion.

1 Freeman D, Pugh K, Antley A, Slater M, Bebbington P, Gittins M, Dunn G, Kuipers E, Fowler D, Garety P. Virtual reality study of paranoid thinking in the general population. Br J Psychiatry 2008; 192: 258-63.

2 Reid WH, Thorne SA. Personality disorders and violence potential. J Psychiatr Pract 2007; 13: 261-8.

3 Hamilton M (ed). Fish's Clinical Psychopathology (2nd edn). ButterworthHeinemann, 1985.

4 Gelder M, Gath D, Mayou R, Cowen P. Oxford Textbook of Psychiatry (2nd edn). Oxford University Press, 1996

5 World Health Organization. The ICD-10 Classification of Mental and Behavioural Disorders: Clinical Descriptions and Diagnostic Guidelines. WHO, 1992.

Richard Braithwaite, Portsmouth City Teaching Primary Care Trust, Cavendish House, 18 Victoria Road, South Southsea, Hampshire PO5 2BZ, UK. Email: richard.braithwaite@ports.nhs.uk

doi: 10.1192/bjp.193.1.81a

Author's reply: All too often the presence of paranoid thinking has only been given significance in relation to diagnosing illness. It has been viewed as a symptom that leads to a diagnosis and that, more or less, is the end of it. An alternative view is that the experience itself should take centre stage. ${ }^{1,2}$ Persecutory thinking is 